\title{
Alterstice
}

Revue internationale de la recherche interculturelle

International Journal of Intercultural Research

Revista International de la Investigacion Intercultural

\section{Enjeux interculturels et politiques}

\section{Yvan Leanza}

Volume 7, numéro 1, 2017

URI : https://id.erudit.org/iderudit/1040605ar

DOI : https://doi.org/10.7202/1040605ar

Aller au sommaire du numéro

Éditeur(s)

Alterstice

ISSN

1923-919X (numérique)

Découvrir la revue

Citer ce document

Leanza, Y. (2017). Enjeux interculturels et politiques. Alterstice, 7(1), 1-2.

https://doi.org/10.7202/1040605ar d'utilisation que vous pouvez consulter en ligne.

https://apropos.erudit.org/fr/usagers/politique-dutilisation/ 


\title{
ÉDITORIAL
}

\section{Enjeux interculturels et politiques}

\author{
Yvan Leanza $^{1}$
}

Les chercheurs qui se définissent comme interculturalistes sont-ils aveugles à la dimension politique du comportement humain et de la vie en société ? La recherche interculturelle, qui prend la culture au sérieux, est-elle intrinsèquement et pathologiquement apolitique ? Je me trouvais récemment dans un congrès, assistant à un symposium sur les questions interculturelles en travail social (ce n'était pas le thème central du congrès) lorsque, après les présentations, une personne dans l'assistance y est allée de son commentaire estimant que lorsque la recherche se penche sur la culture et les différences, sur l'altérité, elle passe à côté de la dimension politique, et elle manque les enjeux centraux de l'inégalité et des rapports dominants-dominés.

C'est un reproche que j'ai souvent entendu, et qui est répété à l'envi depuis plusieurs décennies. Implicitement, ce commentaire relègue les questions interculturelles à un niveau secondaire par rapport à d'autres "vraies » questions. On peut aussi entendre que les interculturalistes sont des naïfs qui ne comprennent pas les «vrais » enjeux. Et, pour finir, une telle affirmation met en opposition le politique et les questions interculturelles. Bien entendu, en réduisant la recherche interculturelle aux seuls enjeux de relations entre personnes de cultures différentes, le reproche ignore tout un pan de ce champ que sont les recherches comparatives (malheureusement bien peu représentées dans cette revue).

Dissocier la dimension interculturelle des autres "vraies questions » est un problème en soi. Pourquoi cette dimension du comportement humain serait-elle secondaire ? L'effort collectif (et individuel) de construction de sens est-il une frivolité ? Ainsi, tenir une telle posture est un choix politique en soi, celui de ne voir que des enjeux politiques (entendez socio-économiques) dans toutes les relations entre humains. C'est aussi réducteur que d'accuser la recherche interculturelle d'oublier la dimension politique! Sous-entendre que les interculturalistes sont des naïfs est condescendant. II s'agit d'une communauté hétérogène, qui a des racines dans diverses disciplines, qui manipule divers cadres théoriques et méthodes, qui inclut nécessairement des éléments critiques, et qui fait preuve de rigueur et de créativité, comme en témoignent deux fois par an les articles d'Alterstice. Cette communauté a su se renouveler et prend en compte ce reproche depuis longtemps, par exemple en développant des réflexions sur le thème "genres, cultures et migrations ", un thème central lors du dernier congrès de l'ARIC.

Continuer à opposer les enjeux interculturels et ceux du politique est probablement une erreur épistémologique : celle de perpétuer une division qui empêche la progression de la connaissance et le développement de pratiques et politiques plus inclusives. II me semble que les rédacteurs invités de ce numéro, Christian Agbobli (UQAM), Bob White (Université de Montréal) et Danielle Gratton (Université de Montréal et Centre intégré de santé et de services sociaux de Laval) vont justement à l'encontre de cette division, développant une démarche qui inclut à la fois les dimensions interculturelles (dynamiques d'interactions en institution) et politiques (processus de discrimination).

$$
* * *
$$


Lors du congrès de I'ARIC au mois de mai de cette année à Antananarivo (voir la Rubriqu'ARIC de ce numéro), le prix pour la meilleure thèse en recherche interculturelle a été attribué à Audrey Heine pour son travail intitulé Entre pays d'origine et pays d'accueil. Les enjeux identitaires de la (non) reconnaissance des descendant-e-s d'immigré-e-s au pays d'origine. Mme Heine a soutenu sa thèse en mars 2015 à l'Université libre de Bruxelles (Belgique). Sa recherche, effectuée sous la direction du professeur Laurent Licata, porte sur les relations des personnes issues de l'immigration envers le pays d'origine des parents, un thème très peu investigué à ce jour. Plus précisément, elle s'est posé deux questions : 1) comment les personnes issues de l'immigration perçoivent-elles le pays d'origine? - cette question appelant une sous-question : y voient-elles une source de reconnaissance ou au contraire une source de non-reconnaissance? - 2) Quelles sont les conséquences pour l'identité de cette (non) reconnaissance perçue au pays d'origine?

Dans un premier temps, les représentations de Belgo-Marocaines et Belgo-Marocains et de Belgo-Turques et BelgoTurcs à l'égard du pays d'origine, de ses habitants et des traditions culturelles ont été explorées, gtrâce à plusieurs entretiens et à l'analyse de discussions sur des forums Internet. Ensuite, des études quantitatives ont porté sur les processus identitaires activés par les perceptions du pays d'origine. Mme Heine a analysé l'effet de la nonreconnaissance au pays d'origine et au pays d'accueil dans trois dimensions : (1) l'identification ethnique et au pays d'accueil, (2) l'identification religieuse et (3) la perception des normes de genre (les valeurs liées aux rôles des hommes et des femmes dans la société). Elle a également examiné dans quelle mesure le genre modérait la relation entre ces variables et le rôle des intentions d'intégration au pays d'origine ainsi que le rôle des processus de socialisation familiale en situation d'acculturation.

Trois constats ont pu être posés : (1) une polarisation des représentations du pays d'origine sur la question des valeurs culturelles (valorisation ou critique des valeurs traditionnelles), (2) un déficit de reconnaissance perçu dans le pays d'accueil, mais également dans le pays d'origine et (3) l'impact de la dimension de genre. En effet, les représentations des sociétés d'appartenance et les processus identitaires mis en place sont influencés par les processus de socialisation sexués, exacerbés en situation d'acculturation. Les travaux effectués dans le cadre de cette thèse permettent donc de reconsidérer la situation des personnes issues de l'immigration. Celles-ci ne se trouvent pas seulement au centre de deux instances sociales, la famille immigrée et la société d'installation, mais à l'intersection d'une multiplicité d'espaces de diffusion culturelle, à l'intérieur desquels le pays d'origine occupe un rôle important pour la construction de l'identité.

Chaleureuses félicitations à Mme Heine !

Bonne lecture !

\section{Rattachement de l'auteur \\ ${ }^{1}$ Université Laval, Québec, Canada}

\section{Correspondance}

alterstice@gmail.com

\section{Pour citer cet article}

Leanza, Y. (2017). Enjeux interculturels et politiques [Éditorial]. Alterstice, 7(1), 1-2. 